\title{
Plane-strain mixed-mode near-tip fields in elastic perfectly plastic solids under small-scale yielding conditions
}

\author{
P. DONG* and J. PAN \\ The University of Michigan, Department of Mechanical Engineering and Applied Mechanics, Ann Arbor, \\ Michigan 48109, USA (*Current address: Edison Welding Institute, Columbus, Ohio 43220, USA)
}

Received 2 September 1988; accepted in revised form 9 August 1989

\begin{abstract}
Within the context of the small-strain approach, plane-strain mixed-mode near-tip fields of a stationary crack in an elastic perfectly plastic Mises solid under small-scale yielding conditions are examined by finite element methods. Steady-state stress fields in the immediate vicinity of the crack tip are obtained as the remote loading of the elastic $K$-field increases. Asymptotic crack-tip solutions consisting of constant stress sectors, centered fan sectors, and an elastic sector are then constructed accordingly. The asymptotic crack-tip stress solutions agree well with the numerical results for a whole spectrum of mixed-mode loadings. Our mixed-mode near-tip solution with an elastic sector differs from that of Saka et al. by one (plastic) constant stress sector situated between the elastic sector and the neighbouring fan sector. The effect of the existence of the elastic sector on the near-tip fields is discussed in the light of the computational results. The plastic mixity factor of the near-tip field is given as a function of the elastic mixity factor of the prescribed $K$-field. This function is well bounded by that of the perfectly plastic limit of the corresponding solutions for power-law hardening materials given by Shih. Some issues pertaining to the numerical procedures such as the implementation of the small-scale yielding assumption are also addressed.
\end{abstract}

\section{Introduction}

The asymptotic plane-strain and plane-stress crack-tip fields for both power-law hardening and perfectly plastic materials have been presented by Hutchinson [1, 2], Rice [3] and Rice and Rosengren [4] under pure mode I and pure mode II conditions, and by Shih [5, 6] under mixed-mode conditions. For power-law hardening materials, the asymptotic crack-tip stress and strain fields possess the well-known HRR singularity. The crack-tip field solutions for perfectly plastic materials presented by these authors are constructed with the assumption that the material surrounding the crack tip is fully yielded at all angles. These solutions agree with the perfectly plastic limits of the corresponding asymptotic solutions for power-law hardening materials. Note that these solutions for perfectly plastic materials under planestrain near mode I mixed-mode conditions and under plane-stress mode I and mixed-mode conditions contain radial stress discontinuities (for anisotropic perfectly plastic materials, see Pan [7, 8]).

For elastic perfectly plastic materials, the plane-strain mode I asymptotic crack-tip field (known as the Prandtl field) proposed by Rice [3] and Rice and Rosengren [4] has been verified by finite element computations under small-scale yielding conditions (for example, see Levy et al. [12] and Rice and Tracey [13]). Under mixed-mode loading conditions, Gao [9], with the assumption of Poisson's ratio $v$ being $1 / 2$, constructed theoretically plane-strain crack-tip fields that contain no stress discontinuities, but include two elastic sectors. Gao and Nemat-Nasser [10] and Nemat-Nasser and Obata [1 1] later suggested a mode II solution with 
two elastic sectors. Due to the nonuniqueness nature of the perfect-plasticity solutions, these solutions could be viewed as solutions of many possibilities, and have not been verified by any other means. Later, Saka et al. [14], based on the results of their finite element computations, suggested a different near mode I mixed-mode near-tip structure from Gao's solution [9]. They found that, in reference to Gao's solution [9] valid for $v=1 / 2$, the elastic sector bordered by the upper crack surface indeed exists and the elastic sector bordered by the lower crack surface should be a (plastic) constant stress sector according to their FEM calculations for $M^{p}=0.9,0.79,0.7$, with Poisson's ratio $v$ being nearly $1 / 2$.

In this paper, the plane-strain small-scale yielding near-tip fields under monotonically increasing mixed-mode loading with a fixed mode mixity at the remote elastic boundary are examined using finite element methods. The material is assumed to be an elastic perfectly plastic solid obeying the $J_{2}$ flow theory. Attempts are made in elucidating the details of the limiting stress states near a crack tip, which will be referred to as the steady-stress states. Our accurate finite element computations show that, under the majority of mixed-mode loading conditions, the near-tip field contains an elastic sector but our near-tip field differs from that of Saka et al. [14] by one more (plastic) constant stress sector situated between the elastic sector and the neighbouring fan sector for Poisson's ratio being 0.3 and $0.495(\approx 1 / 2)$. The asymptotic near-tip fields appropriate for elastic perfectly plastic solids are assembled and are shown to be in excellent agreement with the finite element results for a whole spectrum of mixed-mode loadings. The near-tip fields for plane stress will be discussed in a subsequent article [15].

\section{Finite element analysis}

\subsection{Computational model}

We consider a crack in a solid of elastic perfectly plastic material with the coordinate systems depicted in Fig. 1; the Cartesian coordinates $x_{1}$ and $x_{2}$ and the polar coordinates $r$ and $\theta$ are centered at the tip. The mixed-mode small-scale yielding problem was modelled by considering the crack in a circular domain of radius $r_{0}$ as shown. The circular domain was entirely discretized by finite elements. In the immediate crack-tip region we used a ring of 40 wedge-shaped elements of size $r_{i}$. We used collapsed nodes to simulate the $1 / r$ singularity in strain at the tip. The crack-tip elements are equally distributed from $-\pi$ to $\pi$ and surrounded by 24 circular strips of elements generated by a logarithmic scale in the $r$ direction. The entire model consists of 1000 isoparametric elements.

The displacements due to the leading singular terms of the linear elastic asymptotic solution of the crack-tip field,

$$
u_{i}=\frac{1}{2 G} \sqrt{\frac{r}{2 \pi}}\left(K_{1} \hat{u}_{i}(\theta, v)^{\mathrm{I}}+K_{\mathrm{II}} \hat{u}_{i}(\theta, v)^{\mathrm{II}}\right), \quad i=1,2
$$

are specified as the boundary conditions at the outermost boundary $r=r_{0}$ of the domain. Here, $G$ represents the shear modulus, $v$ represents the Poisson ratio, $K_{\mathrm{I}}$ and $K_{\mathrm{II}}$ denote the mode I and mode II stress intensity factors of the far-field, and $\hat{u}_{i}(\theta, v)^{\mathrm{I}}$ and $\hat{u}_{i}(\theta, v)^{\mathrm{Il}}$ are the dimensionless displacement functions associated with the elastic singularity and depend only 


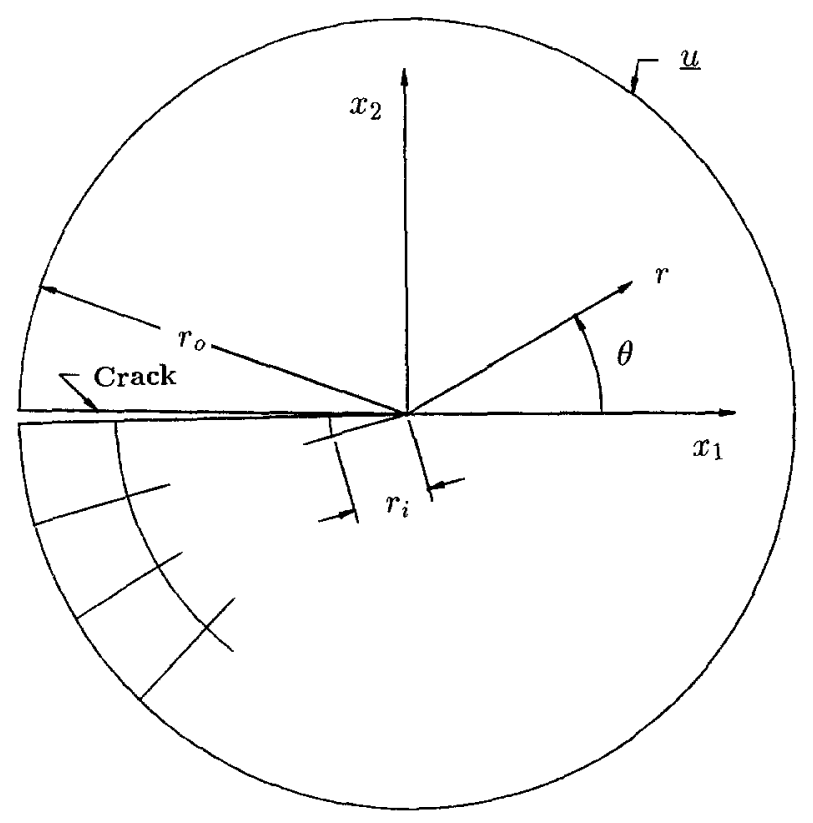

Fig. 1. A finite element model and the coordinate conventions.

on the orientation $\theta$ for a given elastic material. The loading was applied through the stress intensity factors, $K_{1}$ and $K_{\mathrm{II}}$, which are the amplitude factors in (1). The relative composition of $K_{\mathrm{I}}$ and $K_{\mathrm{II}}$ was controlled through a mixity factor $M^{e}$ defined as [5]

$$
\begin{aligned}
M^{e} & =\frac{2}{\pi} \arctan \left[\lim _{r \rightarrow \infty} \frac{\sigma_{00}(r, \theta=0)}{\sigma_{r \theta}(r, \theta=0)}\right] \\
& =\frac{2}{\pi} \arctan \left[\frac{K_{\mathrm{I}}}{K_{\mathrm{II}}}\right],
\end{aligned}
$$

where the magnitude of $M^{e}$ ranges from 0 to 1 , with $M^{e}=0$ for pure mode II, and $M^{e}=1$ for pure mode 1 .

The types of elements used were eight-node serendipity elements and nine-node Lagrangian elements. To relieve artificial mesh-locking that occurs for nearly incompressible materials in plane-strain, we performed a number of numerical experiments on a DEN specimen [16]. The limit load was clearly observed with the eight-node elements using reduced integration and with nine-node elements using the $\bar{B}$-method proposed by Hughs [17]. Due to a large saving of the CPU time, the majority of the numerical results reported here were obtained with eight-node elements using reduced integration, unless otherwise specifically stated.

To test the accuracy of the mesh, we carried out several elastic plastic calculations using fine and coarse meshes. The numerical tests indicated that the results are essentially the same. In order to gain a complete picture of the crack-tip fields we feel that it is necessary to investigate the stress field deeply inside the plastic zone. Therefore, we leave the relative crack-tip element size $r_{i} / r_{0}$ as a variable, typically ranging from $10^{-12}$ to $10^{-4}$ for this investigation. 


\subsection{Numerical procedure}

The material is modelled here as an elastic perfectly plastic solid. A small-strain incremental plasticity theory was employed with the Huber-Von Mises yield condition and the associated flow rule. A displacement-based finite element method with an iterative procedure based on the modified Newton-Raphson method was used in the analysis. The finite element equations were derived from the principle of virtual work. At a time $t+\Delta t$, this takes the form

$$
\int_{A} \sigma_{i j}(t+\Delta t) \delta \varepsilon_{i j} \mathrm{~d} A=\int_{\ell A} T_{i}(t+\Delta t) \delta u_{i} \mathrm{~d} S
$$

where $\sigma_{i j}(t+\Delta t)$ represents the Cauchy stress tensor, which satisfies the equilibrium conditions at time $t+\Delta t$, and $T_{i}(t+\Delta t)$ is the imposed traction vector on the boundary $\partial A$ of the domain $A$. In addition, $\delta u_{i}$ represents the virtual displacement field that vanishes on the part of the boundary where displacements are specified, and $\delta \varepsilon_{i j}$ is the associated small-strain tensor. Here time is used as a convenient variable to represent different loading levels. Linearizing (3) with respect to the equilibrium configuration at time $t$ and introducing the finite element approximation, we obtain the following incremental equilibrium equations in matrix notation:

$$
\boldsymbol{K}_{T} \Delta \boldsymbol{U}=\boldsymbol{F}(t+\Delta t)-\boldsymbol{P}(t)
$$

where $\Delta U=U(t+\Delta t)-U(t)$ is the vector of incremental displacements at the nodal points, $\boldsymbol{K}_{T}=\int_{A} \boldsymbol{B}^{T} \boldsymbol{D} \boldsymbol{B} \mathrm{d} \boldsymbol{A}$ is the tangent stiffness matrix corresponding to the configuration at time $t$ ( $\boldsymbol{B}$ is the strain-displacement matrix and $\boldsymbol{D}$ the material constitutive matrix of the elastic plastic material), $\boldsymbol{F}(t+\Delta t)$ is the vector of applied external loads at time $t+\Delta t$, and $\boldsymbol{P}(t)=\int_{A} \boldsymbol{B} \sigma(t) \mathrm{d} A$ is the equivalent force vector of the element stresses at time $t$.

The loading was applied through the mode I and mode II stress intensity factors, which enter the far-field displacement boundary conditions (1). The remote load intensity can be expressed in terms of the well-known $J$ integral [3] as

$$
J=\frac{1-v^{2}}{E}\left(K_{1}^{2}+K_{\mathrm{II}}^{2}\right)
$$

in order to facilitate the subsequent discussions. A small initial load (small $J$ ) is applied so that all elements remain elastic. $J$ is then scaled to cause incipient yielding at a crack-tip element where the stresses are the highest. At this point the value of $J$ is denoted as $J_{0}$. The load is then incrementally increased by a fraction of $J_{0}$. An iterative Newton-Raphson procedure is employed in solving the incremental equilibrium equations (4) for each load increment, such that for the $k$ th equilibrium iteration at time $t+\Delta t$, the Euclidean norm

$$
\Delta \boldsymbol{R}^{k}=\boldsymbol{F}(t+\Delta t)-\boldsymbol{P}^{k-1}(t+\Delta t)
$$

satisfies

$$
\frac{\left\|\Delta \boldsymbol{R}^{k}\right\|}{\|\boldsymbol{F}\|} \leqslant T O L
$$


where the TOL is a small preset tolerance. For the problems considered here, the TOL must be carefully controlled in order to obtain accurate crack-tip stress fields. Common tolerance settings are not good enough, and our numerical experiments show that much smaller TOL is needed as $r_{i} / r_{0}$ becomes small. For instance, $T O L=10^{-8}$ was used for $r_{i} / r_{0}=10^{-11}$, and $T O L=10^{-5}$ for $r_{i} / r_{0}=10^{-4}$. It is important that the stress evaluation, which can be written as

$$
\sigma_{i j}^{k}(t+\Delta t)=\sigma_{i j}(t)+\int_{\varepsilon_{i j}(t)}^{k_{i j}^{k}(t+\Delta t)} D_{i j k l} \mathrm{~d} \varepsilon_{k l}
$$

is performed by integrating from the values of the last accepted equilibrium state to the current state of iteration $k$, so that the final results are not affected by the errors introduced in the intermediate iterations [18]. The incremental processes are continued until a steady stress state at the crack tip is observed. At all times the maximum extent of the plastic zone around the crack tip is smaller than $1 / 30$ of $r_{0}$ to preserve the small-scale yielding conditions. For the results reported here, Poisson's ratio $v$ is taken as 0.3 , and the ratio of Young's modulus $E$ to the tensile yield stress $\sigma_{0}, E / \mathrm{s}_{0}$, as 500 .

\subsection{Numerical results}

\subsubsection{Pure mode I}

In the case of elastic perfectly plastic isotropic materials under mode I loading, Rice [3] proposed the Prandtl slip-line field as the near-tip field under small-scale yielding conditions. The solid lines in Fig. 2a show the crack-tip stress field according to the Prandtl field; the symbols are the FEM results taken directly from the Gauss quadrature points in the immediate vicinity of the crack tip at $r / r_{p, \max } \approx 10^{-2}$, after the steady stress state had been observed. Here, $r_{p, \max }$ represents the maximum extent of the plastic zone from the crack tip. In this figure, $\sigma_{r r}, \sigma_{r 0}$, and $\sigma_{\theta \theta}$ are the polar coordinate representations of the stress components, and $\bar{\sigma}$ is the effective stress. Note that the stress components shown in all figures in this paper are normalized by the yield stress $\sigma_{0}$. The stress field obtained from the FEM analysis is exactly as predicted by the Prandtl solution. Of course, this has been verified by Levy et al. [12]. We include this case here for completeness.

\subsubsection{Pure mode II}

For mode II, both our numerical results and the slip-line solution given by Hutchinson [2] are shown in Fig. 2b. To our best knowledge, this is the first finite element solution of its kind. The slip-line stress field is indeed achieved at the crack tip, and the finite element results at $r / r_{p, \max } \approx 10^{-2}$, without using any smoothing techniques, coincide precisely with the slip-line solution. Special attention should be directed to this case. The numerical results shown in Fig. 2b were obtained at the $40 \mathrm{th}$ load increment. The exact same solution, plastically deformed all around the crack tip, had been observed after 25 load increments, and for the subsequent loading the stress field remained the same. No elastic sectors around the crack tip were identified, in contrast to the proposed mode II solution with two elastic sectors by Nemat-Nasser and Obata [11].

\subsubsection{Mixed-modes}

The crack-tip stress fields at $r / r_{p, \max } \approx 10^{-2}$ corresponding to three combinations of $K_{1}$ and $K_{\mathrm{II}}$ of the far-field $\left(M^{e}=0.84,0.7\right.$, and 0.54 ) are shown in Fig. 3 (only every other data point 


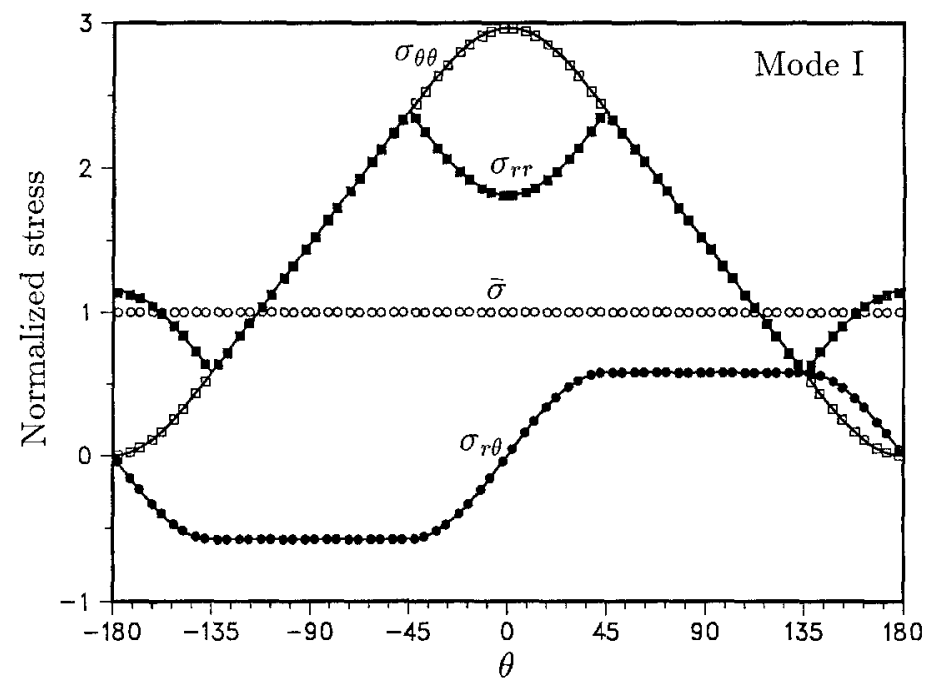

(a)

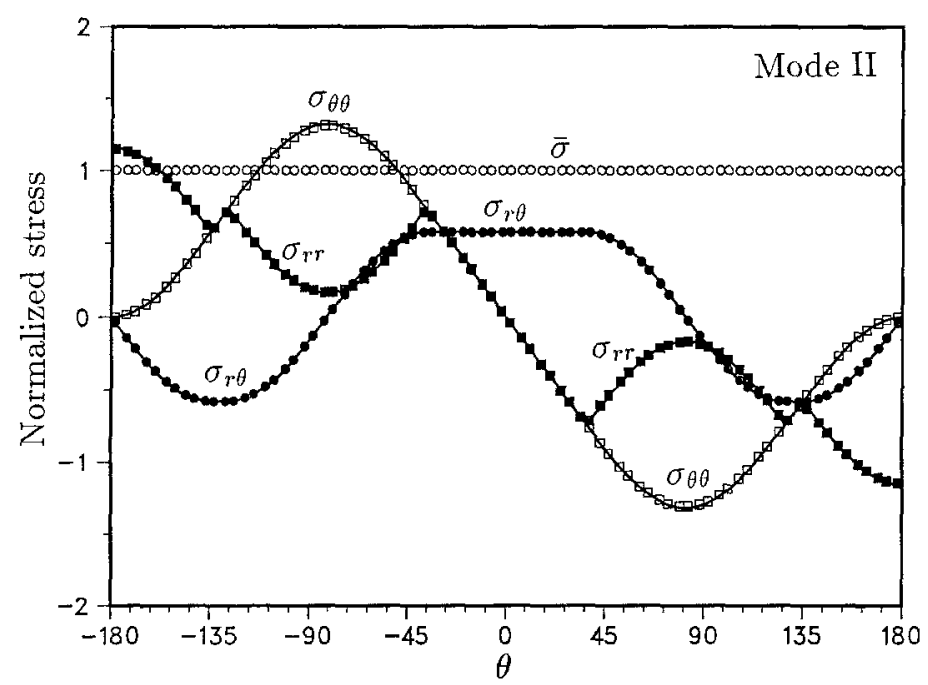

(b)

Fig. 2. Comparisons between the FEM results at $r / r_{p, \max } \approx 10^{-2}$ and the slip-line solutions: (a) mode I, (b) mode II.

is plotted for clarity). The stress field in all cases contain no discontinuity of the radial stress $\sigma_{r r}$, in contrast to the slip-line solutions proposed by Shih [5] where the solutions were constructed with the assumption that the material surrounding the crack tip is fully yielded. It is noted that $\bar{\sigma}$ is less than 1 in a part of $\theta>0$ in the neighbourhood of the upper surface of the crack. In addition, a constant stress sector neighbouring the elastic sector can be identified. This constant stress sector connected the elastic sector and a fan sector (characterized by $\sigma_{r r}=\sigma_{00}$ ). This finding differs from the stress fields presented by Saka et al. [14]. Similar computational results are also obtained for $v=0.495 \approx 1 / 2$. These results indicate that the stress distributions do not change noticeably as $v$ is varied. 


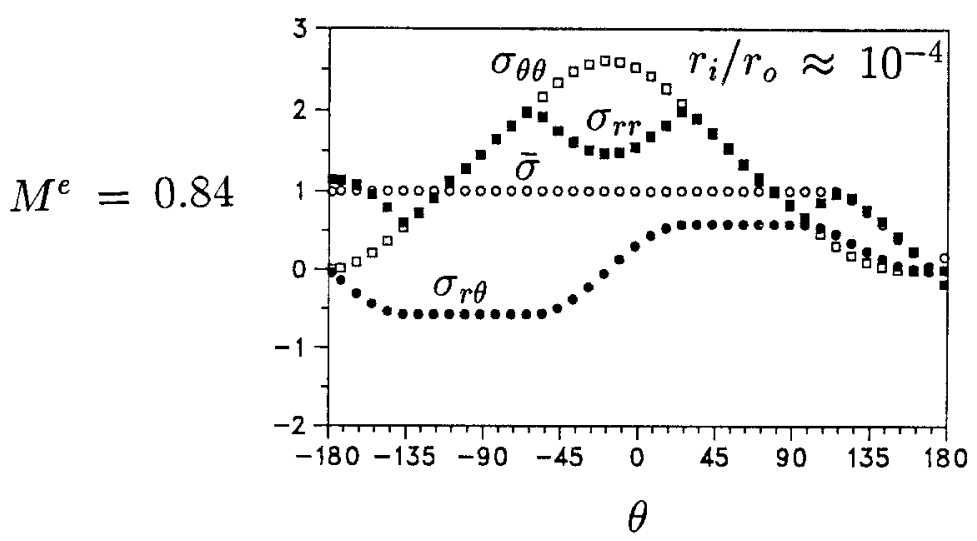

(a)

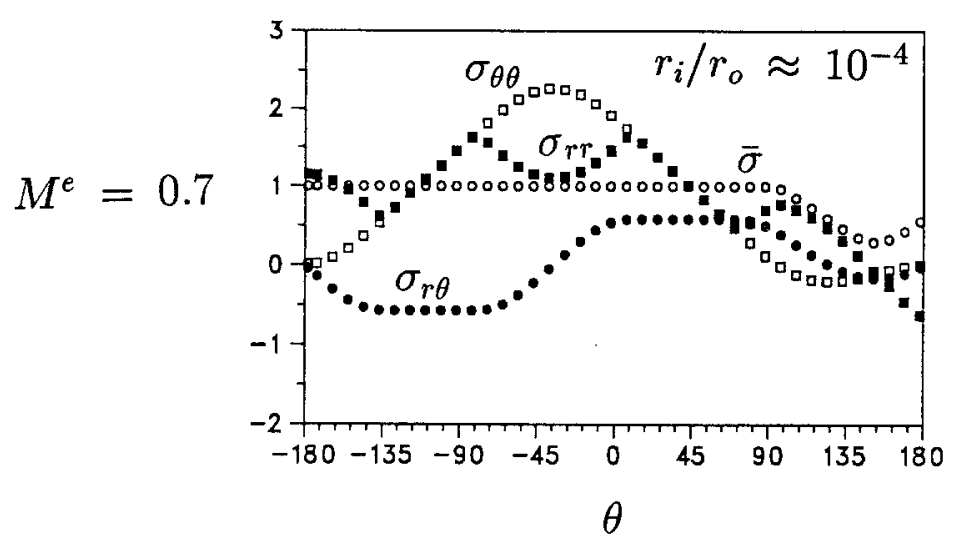

(b)

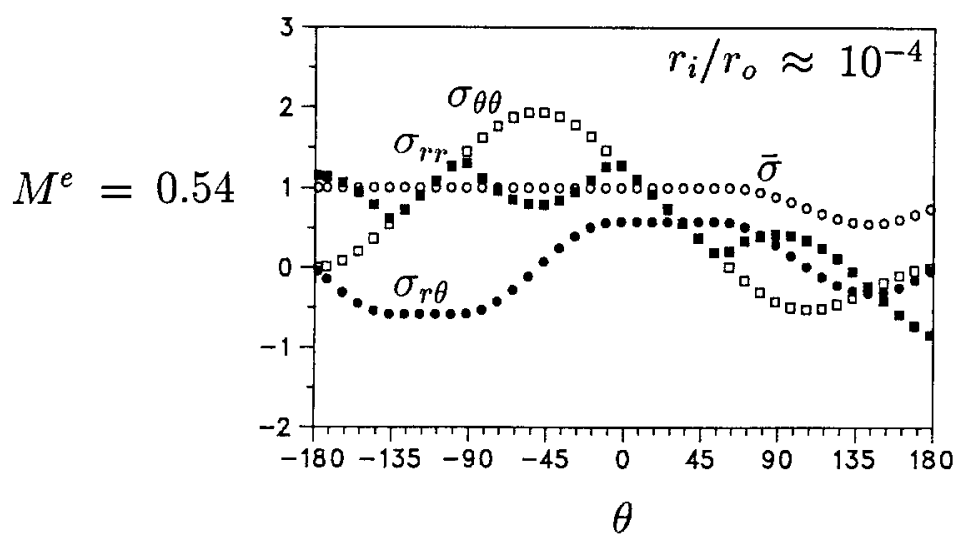

(c)

Fig. 3. Angular variations of the normalized stresses at $r / r_{p, \max } \approx 10^{-2}$ under mixed-mode loadings: (a) $M^{e}=0.84$, (b) $M^{*}=0.7$, and (c) $M^{*}=0.54$. 
These results were obtained along the very first ring of the Gauss quadrature points of the crack-tip elements with $r_{i} / r_{0} \approx 10^{-4}$. Before we generalize the findings and the corresponding stress fields as appropriate representations of the crack-tip stress fields as $r \rightarrow 0$, we further probed the stress fields more closely to the crack tip. This time we used $r_{i} / r_{0} \approx 10^{-11}$ with 28 elements in the $\theta$ direction and 35 in the $r$ direction. This numerical experiment with such a small crack-tip element size allowed us to pursue the mathematical structure of the crack-tip fields. This model also enabled us to impose the remote loading to the highest possible level without violating the small-scale yielding assumption.

The stress fields at two widely separated load levels, measured by $J / J_{0}$, are shown in Fig. 4 for $M^{e}=0.84$. The stress field at $r / r_{p, \max } \approx 2.5 \times 10^{-3}$ shown in Fig. 4 a was obtained at $J / J_{0}=167$ long after a steady stress state at the tip was observed. It clearly gives the same

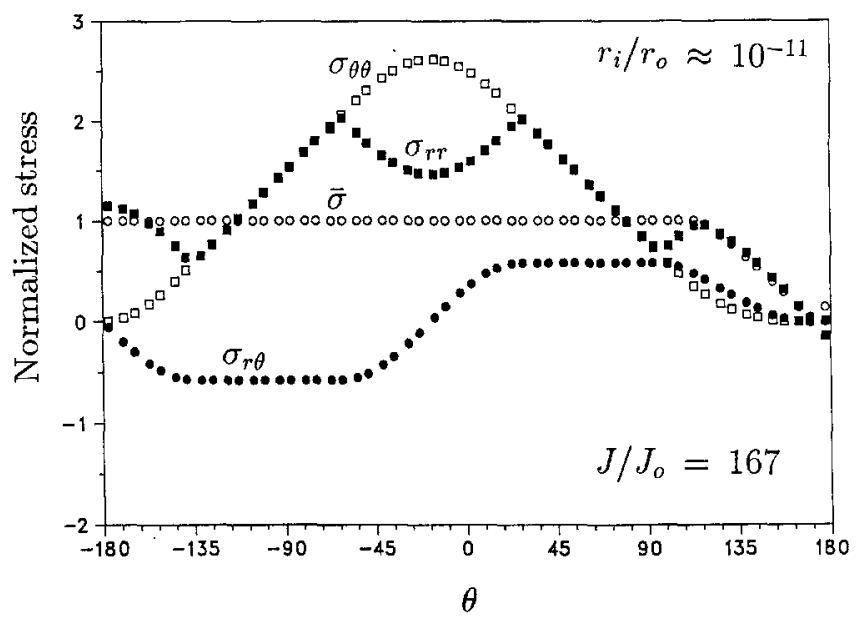

(a)

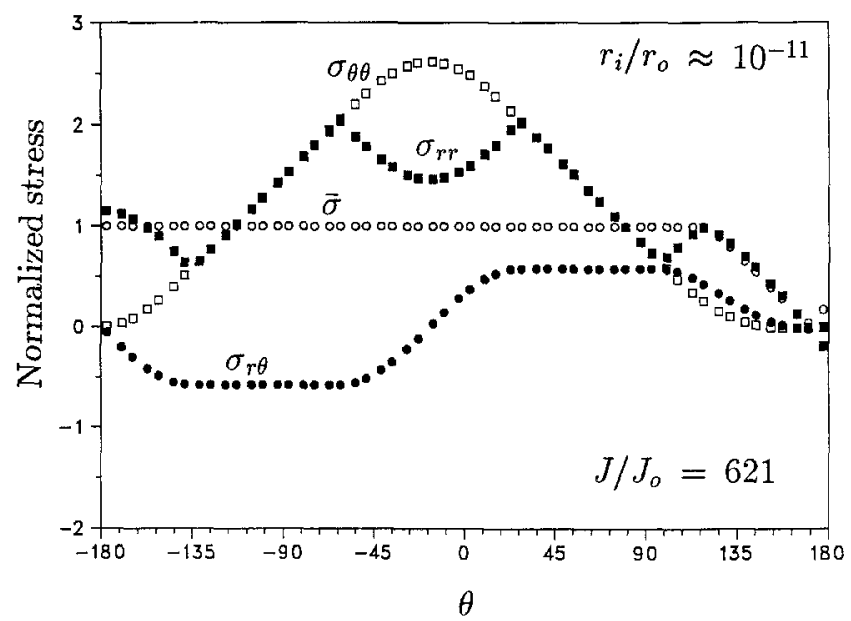

(b)

Fig. 4. Comparisons of the crack-tip stress fields at two widely separated load levels for $M^{*}=0.84$ with a crack-tip element size of $r_{i} / r_{0} \approx 10^{-11}$ : (a) $J / J_{0}=167\left(r / r_{p, \text { max }} \approx 2.5 \times 10^{-3}\right)$, (b) $J / J_{0}=621\left(r / r_{p, \max } \approx 6.7 \times 10^{-4}\right)$. 
stress field as shown in Fig. 3a, where $r_{i} / r_{0} \approx 10^{-4}$ was used. To examine the effect of load intensities, the incremental process was continued to a very high load level. The stress field subjected to $J / J_{0}=621 \mathrm{r} / \mathrm{r}_{p, \max } \approx 6.7 \times 10^{-4}$ is shown in Fig. $4 \mathrm{~b}$. When we compare Figs. $4 \mathrm{a}$ and $4 \mathrm{~b}$, there is no noticeable difference in the stress fields: all the stress components are continuous, and an elastic sector exists.

In Figs. 5a and 5b, we plot the normalized stresses with respect to the normalized radial distance from the crack tip $\left(r / r_{p}\right)$ in a logarithmic scale along two radial lines for the case of $M^{e}=0.84$ at the highest load level permitted by our computational facilities. One radial line

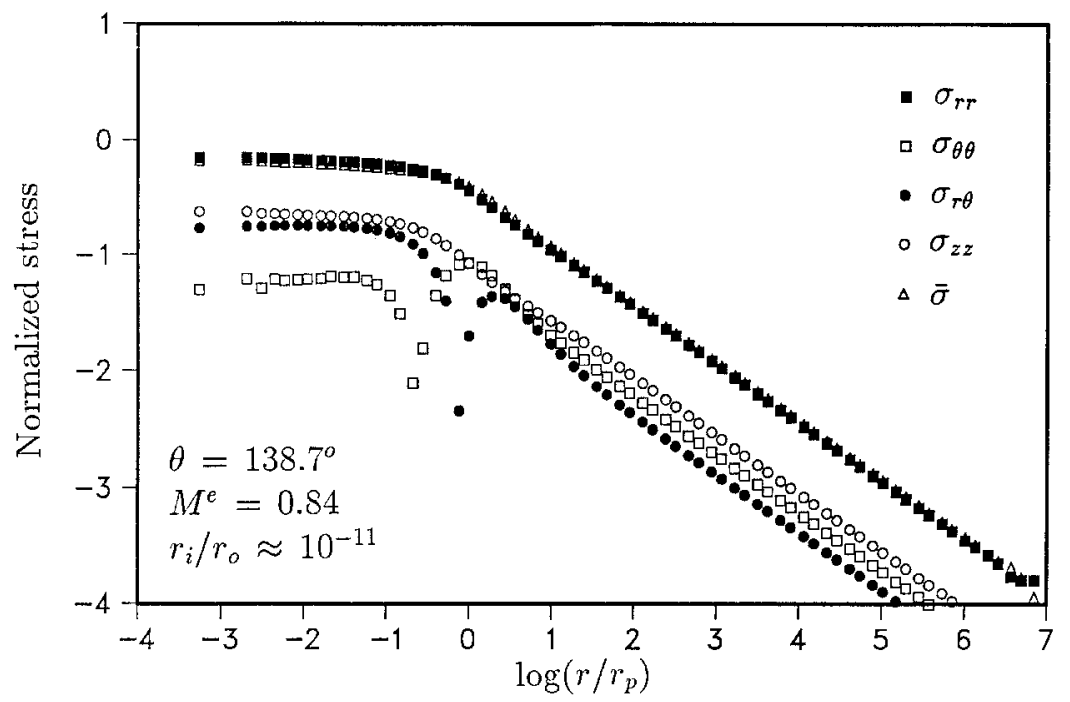

(a)

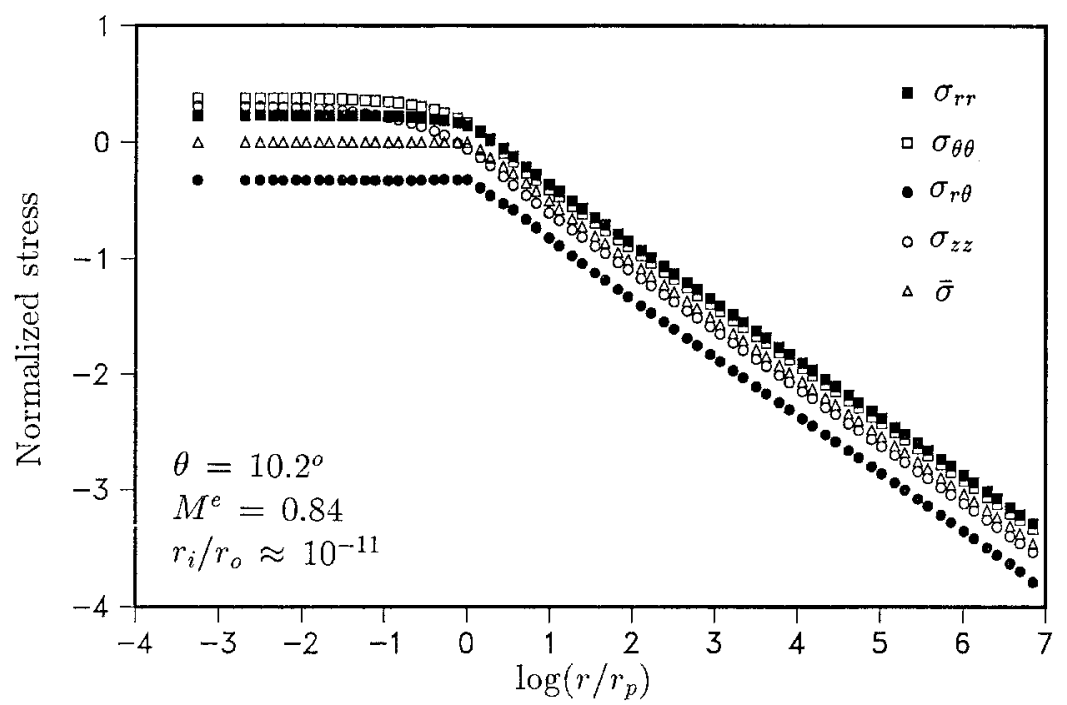

(b)

Fig. 5. Log-log plots of the normalized stresses as functions of the normalized radial distance for $M^{\prime}=0.84$ at two angles: (a) $\theta=138.7^{\circ}$ (inside the elastic region), (b) $\theta=10.2^{\circ}$ (inside the plastically deformed region). 
is within the elastic sector at $\theta=138.7^{\circ}$, and the other is well inside the plastically deformed region at $\theta=10.2^{\circ}$. Note that $r_{p}$ represents the plastic zone size at $\theta=0$. In both figures, we see that for $r / r_{p}>1$, the straight line distributions of the normalized stresses as functions of the normalized radial distance have a slope of exactly $-1 / 2$ and clearly indicate the $r^{-1 / 2}$ singularity as it must be in the elastic $K$-field. As $r / r_{p}$ approaches 1 , the magnitude of the slope decreases. As $r / r_{p}$ decreases from 1, the slope of these lines becomes exactly zero well inside the plastically deformed region (Fig. 5b) and becomes approximately zero within the elastic sector (Fig. 5a).

Similar results to those shown in Figs. 5a and 5b are also observed for $M^{e}=\underline{0.7}$ and 0.54 . This indicates that the stresses are nonsingular within both the plastic sectors and the elastic sector. Therefore, the trend indicates that the stress field at smaller $r / r_{p}$ would be the same as those presented in Fig. 3, and that the stress fields shown in Fig. 3 may be taken as the correct representations of the small-scale yielding mixed-mode near-tip stress fields as $r \rightarrow 0$.

\section{Asymptotic near-tip fields}

\subsection{Equilibrium equations}

In this and subsequent sections, we intend to seek a near-tip solution in which all the stresses are continuous. With reference to the polar coordinates (see Fig. 1), the equilibrium equations can be written as

$$
\begin{aligned}
& \frac{\partial \sigma_{r r}}{\partial r}+\frac{1}{r} \frac{\partial \sigma_{r 0}}{\partial \theta}+\frac{\sigma_{r r}-\sigma_{00}}{r}=0, \\
& \frac{1}{r} \frac{\partial \sigma_{00}}{\partial \theta}+\frac{\partial \sigma_{r \theta}}{\partial r}+\frac{2 \sigma_{r 0}}{r}=0 .
\end{aligned}
$$

Rice $[3,20]$ analyzed the stress state $\sigma_{i j}=\sigma_{i j}(\theta)$ as $r \rightarrow 0$ at the tip of a stationary and a growing crack in an elastic perfectly plastic solid. He argued that since the stress at the tip must be bounded, terms of the form $r\left(\partial \sigma_{i j} / \partial r\right)$ in the equilibrium equations must vanish as $r \rightarrow 0$. Hence (9) and (10) reduce to the two ordinary differential equations:

$$
\begin{aligned}
& \sigma_{r r}-\sigma_{\theta 0}+\frac{\mathrm{d} \sigma_{r \theta}}{\mathrm{d} \theta}=0, \\
& 2 \sigma_{r \theta}+\frac{\mathrm{d} \sigma_{\theta 0}}{\mathrm{~d} \theta}=0 .
\end{aligned}
$$

\subsection{Yield criterion}

Following the development of Rice [19], we write the $J_{2}$ yield condition as

$$
\Phi\left(\sigma_{i j}\right)=\frac{3}{2} s_{i j} s_{i j}-\sigma_{0}^{2}=0
$$


where $s_{i j}$ is the deviatoric part of $\sigma_{i j}$. The differential form of the yield condition, as $r$ approaches 0 , is

$$
s_{i j} \frac{\mathrm{d} \sigma_{i j}}{\mathrm{~d} \theta}=0
$$

Under plane-strain conditions, (14), in combination with the equilibrium equations, (11) and (12), gives

$$
\frac{\mathrm{d}\left(\sigma_{11}+\sigma_{22}\right)}{\mathrm{d} \theta} s_{r r}+\frac{\mathrm{d} \sigma_{33}}{\mathrm{~d} \theta} s_{33}=0
$$

\subsection{Plastically deformed region}

Within the context of the small-strain approach, the strain rate tensor is defined as the symmetric part of the velocity gradients:

$$
\dot{\varepsilon}_{i j}=\frac{1}{2}\left(\frac{\partial \dot{u}_{i}}{\partial x_{j}}+\frac{\partial \dot{u}_{j}}{\partial x_{i}}\right) .
$$

For elastic plastic materials, the strain rate tensor can be decomposed into the elastic part $\dot{\varepsilon}_{i j}^{e}$ and the plastic part $\dot{\varepsilon}_{i j}^{p}$ :

$$
\dot{\varepsilon}_{i j}=\dot{\dot{\varepsilon}}_{i j}^{e}+\dot{\varepsilon}_{i j}^{p}
$$

The elastic part, $\dot{\varepsilon}_{i j}^{e}$, is given by

$$
\dot{\varepsilon}_{i j}^{e}=M_{i j k l} \dot{\sigma}_{k l}
$$

where $M_{i j k l}$ is the elastic compliance tensor. The plastic strain rate $\dot{\varepsilon}_{i j}^{p}$ is given by

$$
\dot{\varepsilon}_{i j}^{p}=\dot{\lambda} s_{i j}
$$

where $\dot{\lambda}$ is a proportional constant. The plane-strain condition requires

$$
\varepsilon_{33}=\varepsilon_{33}^{e}+\varepsilon_{33}^{p}=M_{33 k l} \sigma_{k l}+\varepsilon_{33}^{p}=0
$$

Since in-plane components of $\dot{\varepsilon}_{i j}^{p}$ can develop singularities at the crack tip, while the plastic strain in the $z$-direction is bounded (since the total strain in the $z$ direction and its elastic portion are bounded), it is evident that

$$
\frac{\dot{\varepsilon}_{33}^{p}}{\sqrt{\dot{\varepsilon}_{\alpha \beta}^{p} \dot{\varepsilon}_{\alpha \beta}^{p}}} \rightarrow 0, \quad \alpha, \beta=1,2
$$


as $r \rightarrow 0$. As a result, from the normality flow rule (19) we have $s_{33}=0$ at $r \rightarrow 0$. It then follows that the yield condition (13) reduces to

$$
\frac{\left(\sigma_{00}-\sigma_{r r}\right)^{2}}{4}+\sigma_{r 0}^{2}=\frac{\sigma_{0}^{2}}{3},
$$

and that (15) becomes

$$
\frac{\mathrm{d}\left(\sigma_{11}+\sigma_{22}\right)}{\mathrm{d} \theta} s_{r r}=0
$$

Equation (23) leads to the following forms of the solutions within the plastic region at the crack tip:

(i) Constant stress sectors, in which stresses $\sigma_{11}, \sigma_{22}$, and $\sigma_{12}$ (referred to the Cartesian coordinates) are independent of $\theta$, i.e.,

$$
\begin{aligned}
& \sigma_{11}=\text { constant, } \\
& \sigma_{22}=\text { constant, } \\
& \sigma_{12}=\text { constant, }
\end{aligned}
$$

where the constants are chosen to satisfy the yield condition and other boundary conditions.

(ii) Centered fan sectors, in which $s_{r r}=0$, and

$$
\begin{aligned}
& \sigma_{r 0}= \pm \tau_{0}, \\
& \sigma_{r r}=\sigma_{\theta 0}=\text { constant } \pm 2 \tau_{0} \theta,
\end{aligned}
$$

where $\tau_{0}$ is the yield stress in shear.

\subsection{Elastically deformed region}

As seen in the finite element results, it seems reasonable to admit the existence of an elastic region around the crack tip and to assume that the stresses in the elastic region are nonsingular. For the elastically deformed region, the compatibility equation in terms of stress components gives

$$
\nabla^{2}\left(\sigma_{r r}+\sigma_{00}\right)=0
$$

where $\nabla^{2}$ is the Laplace operator with respect to the polar coordinate system, i.e.,

$$
\nabla^{2}=\frac{\partial^{2}}{\partial r^{2}}+\frac{1}{r} \frac{\partial}{\partial r}+\frac{1}{r^{2}} \frac{\partial^{2}}{\partial \theta^{2}},
$$


and its corresponding asymptotic form for bounded stresses as $r \rightarrow 0$ is

$$
r^{2}\left(\frac{\partial^{2}}{\partial r^{2}}+\frac{1}{r} \frac{\partial}{\partial r}+\frac{1}{r^{2} \partial} \frac{\partial^{2}}{\theta^{2}}\right) \rightarrow \frac{\partial^{2}}{\partial \theta^{2}}
$$

Then, the compatibility equation (24) becomes an ordinary differential equation

$$
\frac{\mathrm{d}^{2}}{\mathrm{~d} \theta^{2}}\left(\sigma_{r r}+\sigma_{00}\right)=0
$$

Solving (11), (12), and (27), we can write the solutions for the stress components in the following forms:

$$
\begin{aligned}
& \sigma_{r r}=2 A+2 B \theta-2 C \cos 2 \theta-2 D \sin 2 \theta, \\
& \sigma_{\theta \theta}=2 A+2 B \theta+2 C \cos 2 \theta+2 D \sin 2 \theta, \\
& \sigma_{r \theta}=-B+2 C \sin 2 \theta-2 D \cos 2 \theta .
\end{aligned}
$$

The integration constants $A, B, C$, and $D$ are to be determined from the boundary conditions.

\subsection{Assembly of the crack-tip solutions}

The possible crack-tip sectors categorized in the previous sections may be assembled in a manner that is consistent with the continuity of $\sigma_{r r}, \sigma_{r \theta}$, and $\sigma_{\theta 0}$, since we seek a solution in which all the stresses are continuous. (Note that the traction continuity across a radial line emanating from the crack tip requires the continuity of $\sigma_{\theta \theta}$ and $\sigma_{r \theta}$, but not $\sigma_{r r}$.) For near mode I mixed-mode loadings, the appropriate form of the solution suggested by the FEM results is shown in Fig. 6 where regions (1), (3), and (5) are the plastic constant stress sectors; (2) and (4) the plastic fan sectors; and (6) the elastic sector. The continuity of all the stresses requires that $\sigma_{r r}, \sigma_{r 0}$, and $\sigma_{00}$ are continuous along the boundaries between two adjacent sectors. In addition, the stresses in regions (1) and (6) must satisfy the boundary conditions on the crack surfaces,

$$
\sigma_{00}( \pm \pi)=\sigma_{r i 0}( \pm \pi)=0
$$

The integration constants for the plastic and elastic regions are obtained by solving simultaneously a system of five equations. For instance, for the elastic sector we have

$$
\begin{aligned}
& A=-\frac{\tau_{0}}{2}\left[\frac{\pi \cos 2 \theta_{4}}{\sin ^{2} \theta_{5}}+\frac{\cos \left(2 \theta_{4}-\theta_{5}\right)}{\sin \theta_{5}}\right], \\
& B=\frac{\tau_{0}}{2} \frac{\cos 2 \theta_{4}}{\sin ^{2} \theta_{5}},
\end{aligned}
$$




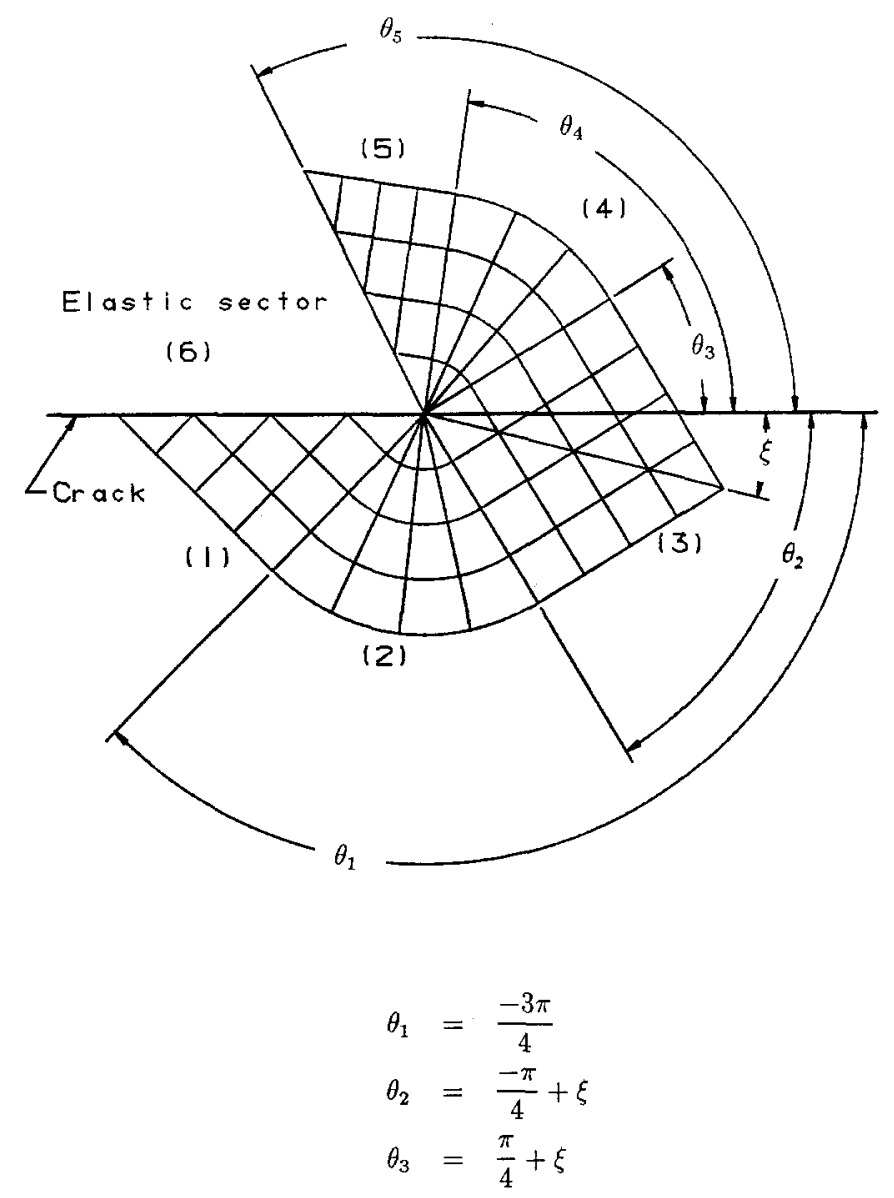

Fig. 6. Assembly of the crack-tip solutions under mixed-mode loading.

$$
\begin{aligned}
& C=\frac{\tau_{0}}{2} \frac{\cos \left(2 \theta_{4}-\theta_{5}\right)}{\sin \theta_{5}}, \\
& D=-\frac{\tau_{0}}{4} \frac{\cos 2 \theta_{4}}{\sin ^{2} \theta_{5}},
\end{aligned}
$$

where $\theta_{4}$ and $\theta_{5}$ satisfy the following equation

$$
\left(\theta_{5}-\pi\right) \frac{\cos 2 \theta_{4}}{\sin ^{2} \theta_{5}}-\frac{\cos \left(2 \theta_{4}-\theta_{5}\right)}{\sin \theta_{5}}=1+4 \xi-2 \theta_{4}+\frac{3 \pi}{2} .
$$

The parameter $\xi$ is a function of the near-field mixity parameter $M^{p}$, defined by Shih [5] in terms of opening and shear stresses ahead of the crack tip as

$$
M^{p}=\frac{2}{\pi} \arctan \left[\lim _{r \rightarrow 0} \frac{\sigma_{00}(r, \theta=0)}{\sigma_{r \theta}(r, \theta=0)}\right]
$$




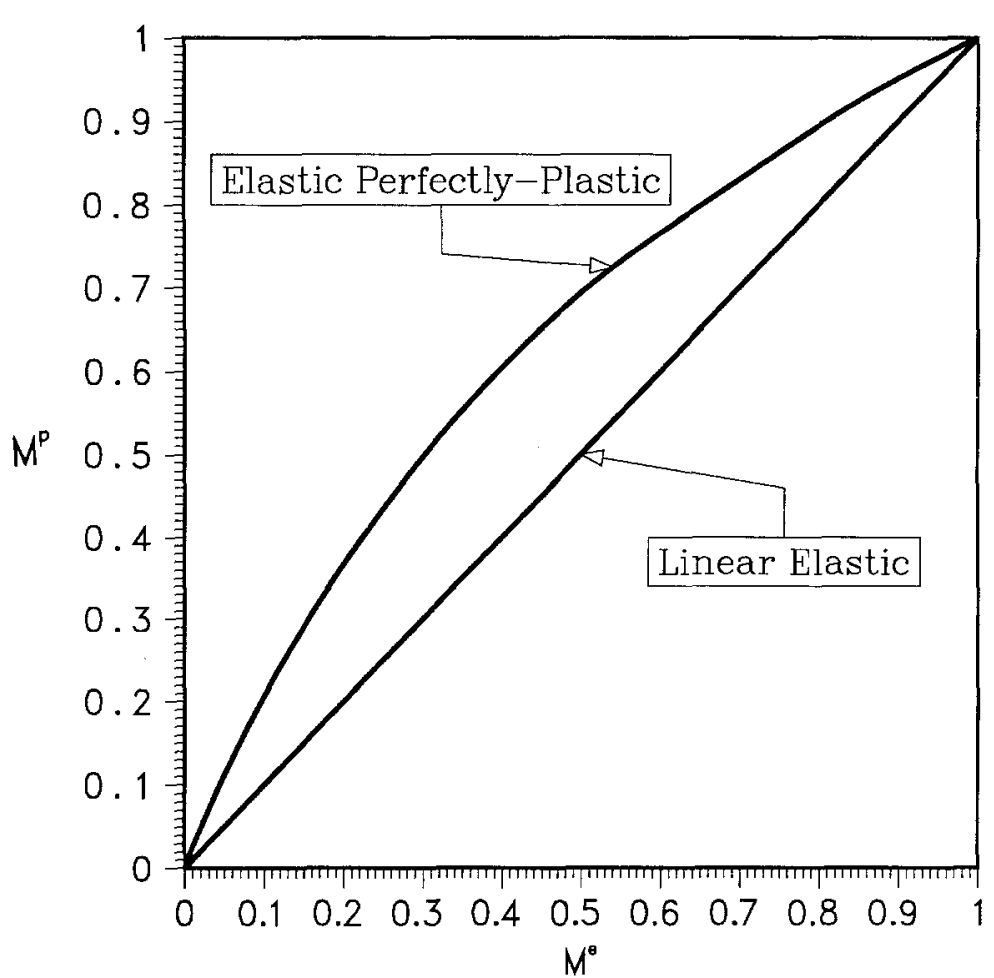

Fig. 7. Relationship between the near-field plastic mixity $M^{p}$ and the far-field elastic mixity $M^{e}$ obtained from the current FEM analysis.

For the slip-line field shown in Fig. 6, $\xi$ and $M^{p}$ can be related as the following

$$
M^{p}= \begin{cases}\frac{2}{\pi} \arctan \left[\frac{1+\pi-2 \xi+\cos 2 \xi}{-\sin 2 \xi}\right], & \text { if }-\frac{\pi}{4} \leqslant \xi \leqslant 0 \\ \frac{2}{\pi} \arctan \left(1+\frac{3 \pi}{2}+4 \xi\right), & \text { if }-\frac{\pi}{4}-\frac{1}{2} \leqslant \xi \leqslant-\frac{\pi}{4}\end{cases}
$$

For a given far-field mixity parameter $M^{e}$, defined by (2), there exists a unique value of $M^{p}$ for our monotonically increasing proportional loading of $K_{1}$ and $K_{\mathrm{ll}}$. From the results of our finite element computations, the relationship between $M^{p}$ and $M^{e}$ is plotted in Fig. 7. Examining (36), we can identify two special cases:

(i). $\xi=0$.

This is the case for $M^{p}=1$ (corresponding to mode I loading). From (36), we have

$$
\theta_{4}=\theta_{5}=\frac{3 \pi}{4}
$$

As a result, (32) through (35) become

$$
\begin{aligned}
& A=C=-\frac{\tau_{0}}{2}, \\
& B=D=0 .
\end{aligned}
$$


(a) $\xi=0$

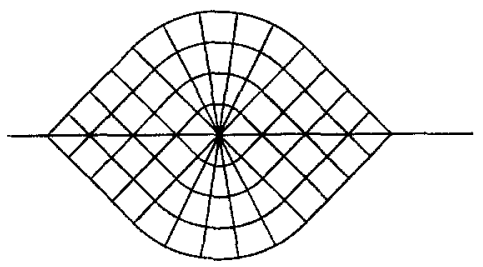

(b)

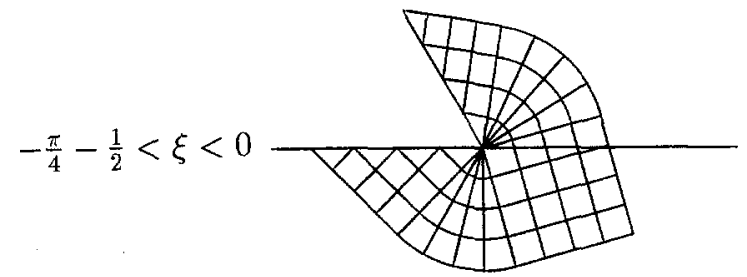

(c) $\xi=-\frac{\pi}{4}-\frac{1}{2}$

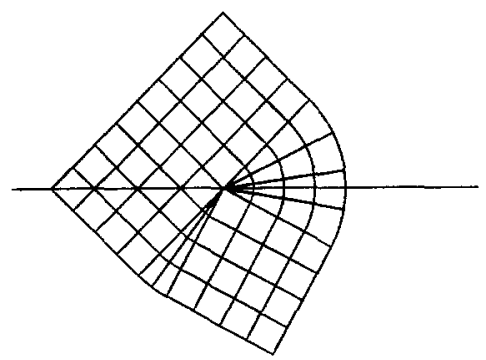

(d) $\xi=-\frac{3 \pi}{8}-\frac{1}{4}$

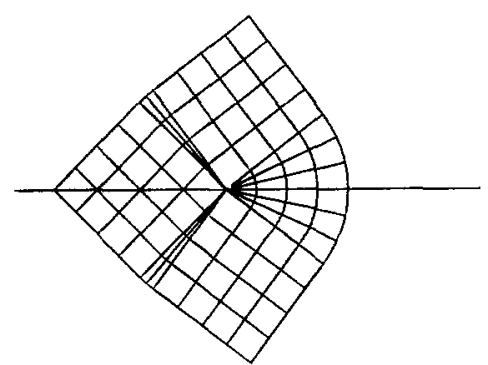

Fig. 8. Typical asymptotic crack-tip fields under mixed-mode loading.

The elastic sector (6) becomes a plastic constant stress sector. The solution corresponds to the Prandtl field proposed by Rice, as shown in Fig. 2a and Fig. 8a.

(ii). $\xi=-\pi / 4-1 / 2$

This is the case for $M^{p}=2 / \pi \arctan (\pi / 2-1) \approx 0.33$. Then, from (36), we have

$$
\theta_{4}=\frac{\pi}{4}, \quad \theta_{5}=\frac{\pi}{4} \text { or } \frac{3 \pi}{4}
$$

Again,

$$
\begin{aligned}
& A=C=-\frac{\tau_{0}}{2} \\
& B=D=0 .
\end{aligned}
$$



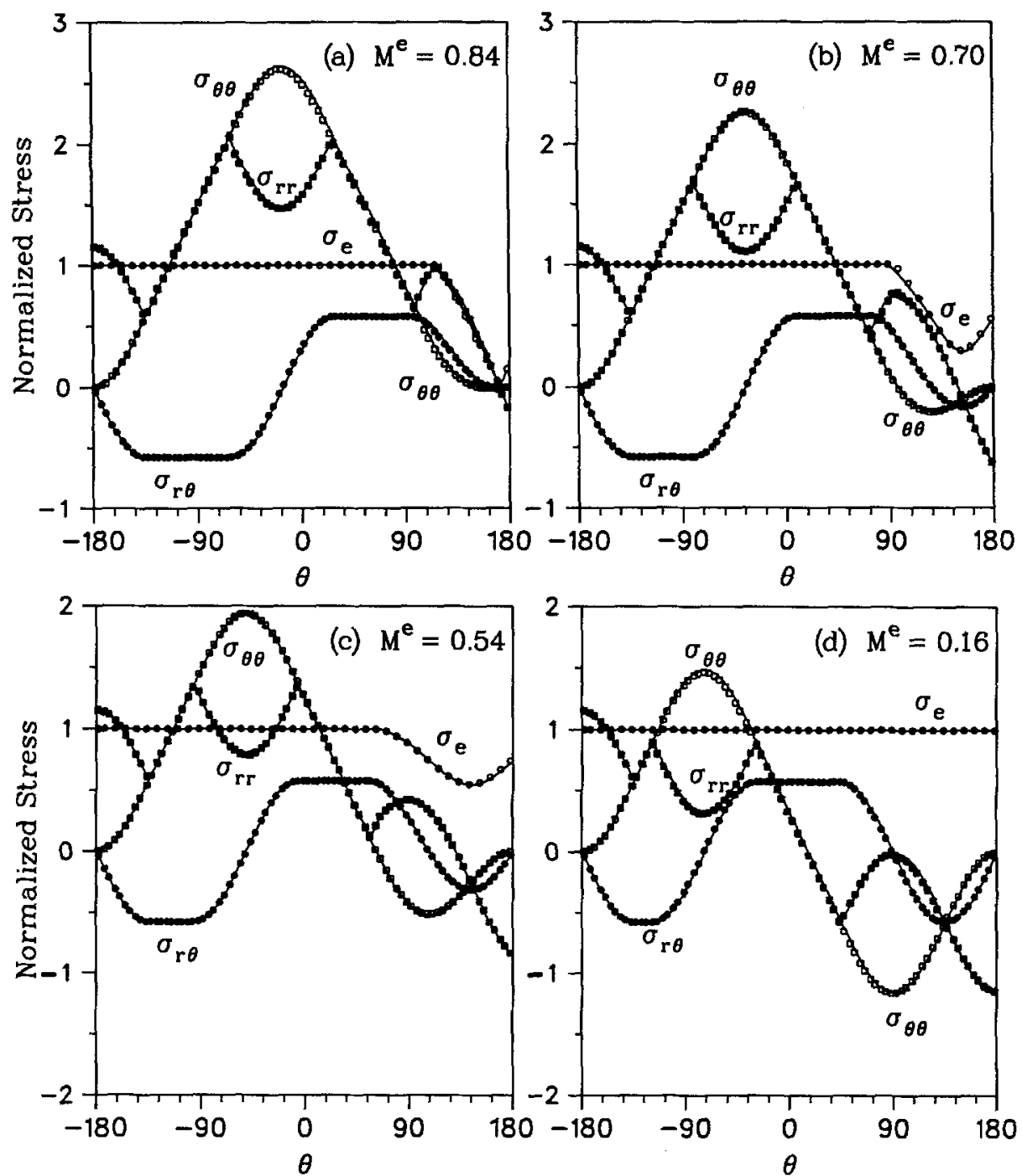

Fig. 9. Comparisons between the FEM results at $r / r_{p, \text { max }} \approx 10^{-2}$ and the current asymptotic solutions: (a) $M^{\prime}=$ 0.84 , (b) $M^{\prime \prime}=0.7$, (c) $M^{\prime \prime}=0.54$, and (d) $M^{\prime \prime}=0$.

As in case (i), the elastic sector (6) becomes a plastic constant stress sector. Plastic yielding occurs at all angles around the crack tip. The corresponding slip-line field is shown in Fig. 8c. Motivated by this development, we performed another finite element calculation using the corresponding far-field mixity parameter $M^{e}=0.16$. The stress fields obtained at $r / r_{p . \max } \approx 10^{-2}$ using the FEM and the slip-line solution are both shown in Fig. 9d. Indeed, plastic yielding all around the crack tip is achieved, and FEM results yield the slip-line solution exactly.

For $-3 \pi / 8-1 / 4 \leqslant \xi<-\pi / 4-1 / 2$, a fan sector develops between regions (5) and (6). All the sectors are plastic. The pure mode II case is shown in Fig. 8d [2]. For $-\pi / 4-1 / 2<\xi<0$, according to (36), region (6), however, remains as an elastic sector (Fig. 8b).

These observations are verified by the FEM solutions at $r / r_{r, \max } \approx 10^{-2}$ shown in Fig. 9 for $M^{e}=0.84,0.7,0.54$, and 0.16 . The solid lines are from the current asymptotic solutions, 
in which $\theta_{5}$ is undetermined from the above analysis for $-\pi / 4-1 / 2<\xi<0$ and the value of $\theta_{5}$ from the FEM solutions is used instead (note that the value of $\theta_{5}$ of the near-tip field for a given elastic mixity does not change noticeably as $v$ was varied; this indicates that the value of $\theta_{5}$ is dominantly determined by material plasticity). The symbols represent the FEM results obtained directly from the Gauss quadrature points of the crack-tip elements. No smoothing techniques were used in any manner. Excellent agreement between the numerical results and the asymptotic solutions is evident. Note that the range of remote mixed-mode loading gives a near-tip field with an elastic sector as shown in Fig. 8b and is quite large (ranging between $M^{e}=0.16$ and 1 ).

\section{Discussion}

As noted in Section 2.3 for the case of $M^{e}=0.84$, the stresses level off within the elastic sector as $r / r_{p}$ approaches 1 , and the slope is approximately zero within the region $r / r_{p}<1$. This is also true for several other mixed-mode cases. Therefore, the asumption that the stress components are not singular in arriving at (28-30) is justified.

If we start with a certain small crack-tip element size, $r_{i} / r_{0}$, and further decrease it, the same steady stress fields result as the remote $K$ field increases. After the remote $K$ field reaches a certain level, further increases in the remote loading cause no noticeable increases in the stress components in the elastic sector, as well as in all the plastic sectors. If plastic yielding at all angles around the crack tip is not impossible, we can at least say that within the length scale and the loading intensity of our investigation, it seems difficult to obtain fully-yielded crack-tip fields under certain combinations of mode I and mode II loading. The existence of elastic sectors may suggest fundamental differences between the non-hardening limit of the power-law hardening solutions and the elastic perfectly plastic solutions. There are indeed no real reasons why they should match: the governing equations (asymptotic forms) for power-law hardening materials are elliptic, whereas the governing equations in the plastically deformed region for elastic perfectly plastic materials are hyperbolic, and they are problems of two different classes.

From this study it is clear that proper numerical conditionings have to be imposed in the analysis of asymptotic crack-tip fields to ensure the validity of the small-scale yielding assumption, under which meaningful stead-state near-tip fields can be numerically extracted. We define $r_{p, \max }$ as the maximum extent of the plastic zone (not necessarily occurring at $\theta=0$ ). When we plotted the mixity factor $M^{p}$ of the near-tip stress field as a function of $r_{p, \max } / r_{i}$ and $J / J_{0}$, we found that the mixity factor $M^{p}$ reaches a steady value at certain values of $r_{p \text { max }} / r_{i}$ and $J / J_{0}$. Further, when we plotted the plastic zones in the normalized coordinates, we found that beyond certain values of $r_{p, \max } / r_{i}$ and $J / J_{0}$, the sizes and the shapes of the plastic zones do not change. Therefore, we may suggest that the fully developed plastic zone be approximately characterized by the parameter

$$
\frac{r_{p, \max }}{r_{i}} \approx 10^{2}
$$

and in terms of remote loading,

$$
\frac{J}{J_{0}} \approx 10^{2}
$$


according to the numerical analyses for all cases of mixed-mode loading. From this point on, since the small-scale yielding condition requires that $r_{p, \text { max }} / r_{0}$ be small, our numerical results show that the self-similarity of the solution can be clearly observed with the upper limit

$$
\frac{r_{p, \max }}{r_{0}} \approx 10^{-2}
$$

This appears to suggest an upper limit of the crack tip element size as $r_{i} / r_{0} \approx 10^{-4}$ for elastic perfectly plastic materials under small-scale yielding conditions.
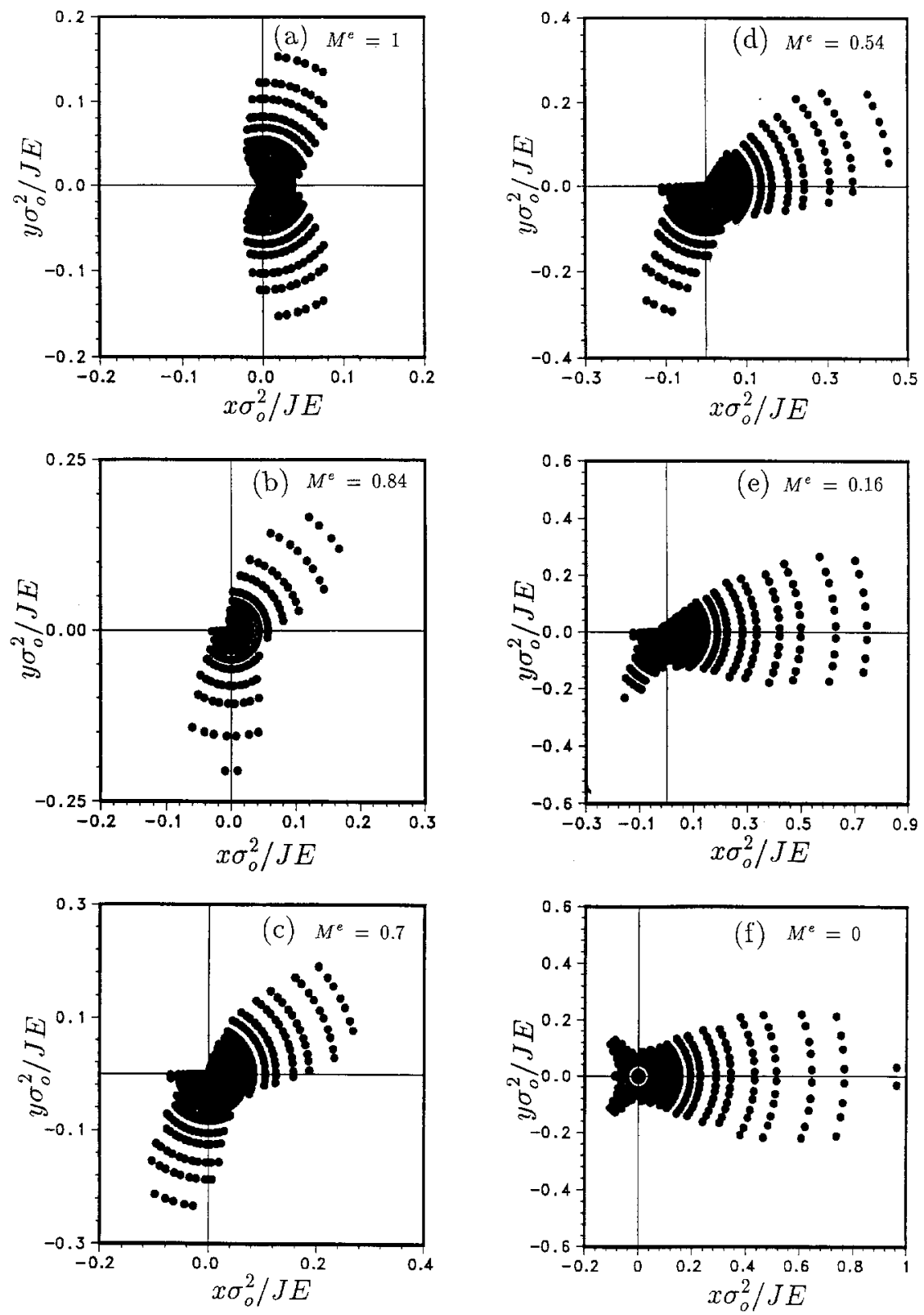

Fig. 10. Plastic zones plotted in the normalized coordinates by $J E / \sigma_{0}^{7}:$ (a) $M^{*}=1$, (b) $M^{*}=0.84$, (c) $M^{e}=0.7$, (d) $M^{\prime}=0.54$, (e) $M^{\prime \prime}=0.16$, and (f) $M^{\prime \prime}=0$. 
The normalized plastic zones are shown in Fig. 10 from the current FEM analysis for pure mode I, pure mode II, and different combinations of remote mixed-mode loadings. A point in the figures represents a Gauss quadrature point in plastic state. The plastic zone sizes are consistently scaled by the self-similar parameter $J E / \sigma_{0}^{2}$ for all the cases, regardless of the remote loading levels. The sizes and the shapes of the plastic zone for elastic perfectly plastic solids are close to those solutions obtained for the power-law hardening material with $n=13$ obtained by Shih [4], even though an elastic sector exists for near mode I mixed-mode loading. For all combinations of the remote mixed-mode loadings, the relationship between the near-field mixity parameter $M^{p}$ and the far-field mixity parameter $M^{e}$ for elastic perfectly plastic solids under the proportional loading of $K_{\mathrm{I}}$ and $K_{\mathrm{II}}$ (see Fig. 7 ) appears to be well bounded by the relationship extrapolated from the power-law hardening solutions by Shih [5].

\section{Acknowledgements}

The authors acknowledge the initial support of this work by the Rackman Faculty Grant of the University of Michigan and the support of the Nuclear Regulatory Commission under grant number NRC-04-87-113. JP also acknowledges the partial support of this work by the National Science Foundation under grant number MSM-8613544. Valuable discussions with the NRC program manager Mike Mayfield and Prof. C.F. Shih of Brown University during the course of this investigation are greatly appreciated.

\section{References}

1. J.W. Hutchinson, Journal of the Mechanics and Physics of Solids 16 (1968) 13-31.

2. J.W. Hutchinson, Journal of the Mechanics and Physics of Solids 16 (1968) 337-347.

3. J.R. Rice, Journal of Applied Mechanics 35 (1968) 379-386.

4. J.R. Rice and G.F. Rosengren, Journal of the Mechanics and Physics of Solids 16 (1968) 1-12.

5. C.F. Shih, Elastic-plastic Analysis of Combined Mode Crack Problems, Ph.D. thesis, Harvard University, Cambridge, Massachusetts (1973).

6. C.F. Shih, in Fracture Analysis, ASTM STP 560 (1974) 187-210.

7. J. Pan, Journal of the Mechanics and Physics of Solids 34 (1986) 617-635.

8. J. Pan, International Journal of Fracture 37 (1988) in press.

9. Y.C. Gao, Acta Solid Mech. Sinica 1 (1980) 69-75 (in Chinese).

10. Y.C. Gao and S. Nemat-Nasser, Journal of the Mechanics and Physics of Solids 32 (1984) 1-19.

11. S. Nemat-Nasser and M. Obata, Mechanics of Materials 3 (1984) 235-243.

12. N. Levy, P.V. Marcal, W.J. Ostergren and J.R. Rice, International Journal of Fracture Mechanics 7, 2 (1971) 143-156.

13. J.R. Rice and D.M. Tracey, in Numerical and Computer Methods in Structural Mechanics, S.J. Fenves et al. (eds.), Academic Press, New York (1973) 585-623.

14. M. Saka, H. Abé and S. Tanaka, Computational Mechanics 1 (1986) 11-19.

15. P. Dong and J. Pan, Plane-stress near-tip fields in elastic perfectly plastic solids under mixed-mode loading conditions, under preparation.

16. P. Dong, Ph.D. thesis, under preparation, The University of Michigan, Ann Arbor (1989).

17. T. Hughs, International Journal of Numerical Methods in Engineering 15 (1980) 1413-1418.

18. K.J. Bathe and A.P. Cimento, Computer Methods in Applied Mechanics and Engineering 22 (1980) 59-85.

19. J.R. Rice, in Mechanics of Solids: The R. Hill 60th Anniversary Volume, H.G. Hopkins and M.J. Sewell (eds.), Pergamon Press, Oxford (1982) 539-562. 